\title{
Performing Personalized Embryo Transfer (Pet) Using the Endometrial Receptivity Analysis (ERA) Test in A Case With Recurrent Implantation Failure
}

\author{
Kaur Jasneet* and Mahajan N \\ Department of Reproductive Medicine. Mother and Child Hospital, Delhi, India
}

Submission: June 20,2018; Published: October 11, 2018

*Corresponding author: Kaur Jasneet, Mother and Child Hospital, Defence Colony, New Delhi, India Tel: 011- 41030715;

E-mail: drjasneet19@gmail.com

\section{Abstract}

Background: Recurrent implantation failure (RIF) is a major challenge to ART success. Despite extensive research a comprehensive understanding of the intricacies of the process of implantation remain elusive. Historically, it has been assumed that the window of implantation is always constant in all women. However, molecular analyses of endometrial receptivity demonstrate a personalized WOI (pWOI) that is displaced in one out of four patients with recurrent implantation failure. Here, we report a clinical case of successful pET after one fresh and three frozen embryo transfer failure.

Case description: A 31-year-old women with primary infertility, grade 4 endometriosis and recurrent implantation failures underwent embryo transfer in the personalized window of implantation as guided by the ERA test and had a successful ART outcome.

Conclusion: In the era of personalized medicine, a "one size fits all" policy is no longer acceptable and performing a personalized embryo transfer in patients with RIF as guided by the ERA test maybe the way forward.

Clinical significance: Diagnosis of endometrial receptivity has posed a challenge because of the lack of accurate, non-invasive, and clinically applicable tests. However, performing the Endometrial Receptivity Analysis (ERA) test which is a customized array based on the transcriptomic signature of human endometrium helps to improve the reproductive outcomes in patients with RIF.

Keywords: Endometrial receptivity analysis; personalized embryo transfer; Recurrent implantation failures

\section{Introduction}

After four decades of IVF, implantation failure still remains a major challenge to ART success. Human implantation is a multifaceted, finely orchestrated event requiring the presence of a healthy embryo, a receptive endometrium, successful embryoendometrial cross talk and maternal immune protection of the allograft [1]. The most important causes of implantation failure include embryo aneuploidy, altered endometrial receptivity and immune dysregulation. Modern tools like genomics and bioinformatics help us in determining the personalized WOI. Our case report shows how performing an embryo transfer in the pWOI can make a difference after several previous IVF attempts.

\section{Case Report}

Mrs S.B. 31-year-old presented to us as a case of primary infertility with grade 4 endometriosis and recurrent implantation failure. She had a married life of 4 years, regular menstrual cycles with previous h/o congestive dysmenorrhea and dyspareunia. She had undergone a laparoscopic ovarian cystectomy along with adhesiolysis in view of chronic pelvic pain three years back. Post her surgery for endometriosis she underwent 4 cycles of ovulation augmentation with both oral ovulogens and gonadotropins followed by 3 cycles of IUI. She underwent two consecutive IVF attempts two years back. In her first cycle she had a fresh embryo transfer in which $2 \mathrm{~d} 3$ embryos were transferred followed by a frozen embryo transfer in which two good quality blastocysts were transferred. In her second IVF cycle a freeze all strategy was employed, and she underwent two frozen embryo transfers, each involving transfer of two good quality blastocysts. However, her urine pregnancy test was however negative.

We planned on another cycle of IVF followed by a frozen embryo transfer (in the pWOI as guided by the ERA test).We followed the flexible antagonist protocol and ovarian stimulation was started with 300 IU recombinant follicle-stimulating hormone (follitropin-alfa Gonal-f®, EMD Serono, Inc.)) guided by her age 31 years ,AMH- $1.9 \mathrm{ng} / \mathrm{ml}$,AFC 5/6 and BMI $24.5 \mathrm{~kg} / \mathrm{m} 2$ for first 5 days followed by Menopur (highly purified HMG-Ferring Pharmaceutical Ltd.) 300 iu for another 4 days. GnRH antagonist (Cetrolix, Intas Pharmaceuticals Ltd.) was started according to the flexible protocol. On day 9 of her stimulation her estradiol levels were $2357 \mathrm{pg} / \mathrm{ml}$ and progesterone level were $0.87 \mathrm{ng} / \mathrm{ml}$ 


\section{Anatomy Physiology \& biochemistry international journal}

with an endometrial thickness of $7.4 \mathrm{~mm}$. Inj human chorionic gonadotropin (hCG) $5000 \mathrm{IU}$ with triptorelin $0.2 \mathrm{mg} \mathrm{s} / \mathrm{c}$ was given as the trigger. Oocyte retrieval was performed 35 hours later under general anesthesia, using transvaginal ultrasound guidance. We retrieved 11 oocytes out of which 9 fertilized and we had $6 \mathrm{~d} 3$ good quality embryos that were cryopreserved.

We performed the endometrial receptivity analysis in the next cycle to determine the personalized window of implantation. ERA was performed in an HRT cycle in which estradiol valerate was started in a dose of $2 \mathrm{mg}$ (after performing a tvs on day 2); was increased to $6 \mathrm{mg}$ till an appropriate endometrial thickness of 7.8 $\mathrm{mm}$ was achieved. This was followed by administration of vaginal progesterone suppository $400 \mathrm{mg}$ twice a day for 5 days starting from day 12 of HRT after ensuring that the serum progesterone was less than $0.9 \mathrm{ng} / \mathrm{ml}$. An endometrial biopsy was performed using the pipelle catheter from Gynetics and the endometrial tissue was transferred to a cryotube containing $1.5 \mathrm{~mL}$ RNA stabilizing agent (Qiagen), vigorously shaken for a few seconds, and kept at $4{ }^{\circ} \mathrm{C}$ in refrigerator for $4 \mathrm{~h}$ which was later transported to the Igenomix lab. ERA result came as post receptive $(p+4.5$ receptive) and it was recommended to perform the blastocyst transfer 12 hours prior to the scheduled time. A downregulated frozen embryo transfer was performed in which two good quality blastocysts were transferred a traumatically under ultrasound guidance as suggested by the ERA test. Luteal phase support in the form of Inj uterine $50 \mathrm{mg}$ daily along with vaginal micronized progesterone $400 \mathrm{mg}$ bd was given. Her urine pregnancy test was positive after 14 days. An ultrasound performed one week later showed a single intrauterine pregnancy with a gestational sac diameter corresponding to $5 \mathrm{w} 2 \mathrm{~d}$.

\section{Discussion}

Recurrent implantation failure remains a major challenge even after four decades of ART. Approximately 10\% of couples undergoing IVF are faced with the issue of RIF. Polanski et al in 2014 [2] suggested that RIF should be defined as the absence of implantation after two consecutive cycles of IVF, ICSI or frozen embryo replacement cycles where the cumulative number of transferred embryos was no less than four for cleavage-stage embryos and no less than two for blastocysts, with all embryos being of good quality and of appropriate developmental stage .Important causes of implantation failure include embryo aneuploidy', altered endometrial receptivity and a dysregulation of the immune system.

Diagnosis of endometrial receptivity has posed a challenge because of the lack of accurate, non-invasive, and clinically applicable tests. Histological, biochemical, and ultrasound markers of ER have been proposed for use to improve implantation rates (IRs) in IVF. Unfortunately, most of these methods are unreliable and do not have any predictive value. However, with the coming up of the ERA test we can have a better insight of the endometrial receptivity. It is a customized array based on the transcriptomic signature of human endometrium and analyzes the expression levels of 248 genes linked to the status of endometrial receptivity, using RNA sequencing with the help of NGS [3]. It involves use of an informatic predictor that analyzes the gene expression data and classifies the endometrium as "Receptive" or "Non-Receptive" with a sensitivity of 0.997 and a specificity of 0.885 and helps us to determine the pWOI [4].

Studies have shown that in patients with endometriosis there is a dysregulation of selected genes in the endometrium leading to impaired embryonic attachment, embryotoxicity and apoptosis during the window of implantation. Several molecules involved in implantation have been shown to be differently expressed in patients with endometriosis including reduced expression of the cellular adhesion molecule $\alpha v \beta 3$ integrin and altered expression of methylation of HOXA10, a potent stimulator of $\alpha \mathrm{v} \beta 3$ expression. However, there is still a lack of conclusive evidence to prove the role of endometriosis in altering the endometrial receptivity [5]. ERA is a step forward in improving ART outcomes by personalizing embryo transfers in patients with RIF. It is the most objective and accurate test available today for diagnosing endometrial receptivity and is extremely beneficial in patients with RIF. Studies have shown that in about 25 percent of patients with RIF there is a displacement in the WOI which could be caused by some intrinsic genomic alterations and so personalizing our embryo transfers might help to improve the reproductive performance in these patients [4].

\section{Conclusion}

In the era of personalized medicine, a "one size fits all" policy is no longer acceptable and performing an embryo transfer in patients with RIF as guided by the ERA test which allows synchronization between the blastocyst and a receptive endometrium - a key factor in promoting implantation; is the way forward.

\section{Acknowledgement}

Nil.

\section{Conflict of interest}

Nil.

\section{References}

1. Coughlan C, Ledger W, Wang Q, Liu F, Demirol A et al (2014) Recurrent implantation failure: definition and management. Reproductive BioMedicine Online 28(1): 14-38.

2. Polanski LT, Baumgarten MN, Quenby S, Brosens J, Campbell BK et al (2014) What exactly do we mean by 'recurrent implantation failure? A systematic review and opinion. Reproductive BioMedicine Online 28(4): 409-23.

3. Mahajan N (2015) Endometrial receptivity array: Clinical application. J Hum Reprod Sci 8(3): 121-9.

4. Díaz Gimeno P, Ruiz-Alonso M, Blesa D, Bosch N, Martínez-Conejero JA, Alamá P et al (2013) The accuracy and reproducibility of the endometrial receptivity array is superior to histology as a diagnostic method for endometrial receptivity. Fertile Sterile 99(2): 508-517.

5. May KE, Villar, J, Kirtley S, Kennedy SH, Becker CM (2011) Endometrial alterations in endometriosis: a systematic review of putative biomarkers. Hum Reprod Update 17: 637-653. 
This work is licensed under Creative Commons Attribution 4.0 License

DOI: 10.19080/APBIJ.2018.05.555666

\section{Your next submission with Juniper Publishers will reach you the below assets}

- Quality Editorial service

- Swift Peer Review

- Reprints availability

- E-prints Service

- Manuscript Podcast for convenient understanding

- Global attainment for your research

- Manuscript accessibility in different formats ( Pdf, E-pub, Full Text, Audio)

- Unceasing customer service

Track the below URL for one-step submission https://juniperpublishers.com/online-submission.php 